\title{
Predictive Markers of Atrial Fibrillation Progression in Heart Failure
}

\author{
Roman E. Tokmachev, $\mathrm{PhD}^{1}$; Andrey Ya. Kravchenko, $\mathrm{PhD}, \mathrm{ScD}^{1}$; \\ Andrey V. Budnevsky, PhD, ScD ${ }^{1}$; Alexei A. Natarov'; Aleksandr I. Zhdanov, PhD, ScD ${ }^{1}$; \\ Evgeniy S. Ovsyannikov, $\mathrm{PhD}^{1^{*}}$; Tatiana A. Chernik ${ }^{1}$; Vyacheslav M. Provotorov, $\mathrm{PhD}, \mathrm{ScD}^{1}$; \\ Nadezhda I. Ostroushko ${ }^{3}$; Julia A. Sharapova ${ }^{3}$ \\ ${ }^{I}$ Voronezh N.N. Burdenko State Medical University \\ ${ }^{2}$ Voronezh City Emergency Hospital №1 \\ ${ }^{3}$ Department of Health of the Voronezh Region \\ Voronezh, the Russian Federation
}

\begin{abstract}
Atrial fibrillation (AF) is one of the most common arrhythmias encountered in clinical practice. It has been found that the presence of chronic heart failure increases the risk of AF by 5 times. Furthermore, with an increase in the severity of symptoms of heart failure, the incidence and severity of AF increases. To date, markers of AF progression are not clearly defined. In this regard, it is relevant and reasonable to identify such markers in patients with heart failure.

The aim of this research was to study the prognostic value of the 6-minute walk test (6MWT), NT-proBNP level, and left ventricular diastolic dysfunction for AF progression in patients with chronic heart failure (CHF).

The study involved 96 participants with stable Class II and III CHF (the NYHA Functional Classification), who were included in the regional registry of CHF patients in September-November 2014. The data obtained showed that in patients with CHF, an increase in the level of Nt-proBNP, impaired myocardial relaxation, and a decrease in 6MWT distance may serve as predictors of AF progression with the transition of arrhythmias to stable forms. (International Journal of Biomedicine. 2020;10(1):20-23.)
\end{abstract}

Key Words: atrial fibrillation $\bullet$ NT-proBNP • 6-minute walking test $\bullet$ diastolic dysfunction

\section{Abbreviations}

6MWT, the six-minute walk test; AF, atrial fibrillation; CHF, chronic heart failure; IVST, interventricular septal thickness; LA, left atrium; LVEF, left ventricular ejection fraction; LVDD, left ventricular diastolic dysfunction; LVESD, left ventricular endsystolic dimension; LVEDD, left ventricular end-diastolic dimension; LVPWT, left ventricular posterior wall thickness.

\section{Introduction}

Atrial fibrillation (AF) is one of the most common arrhythmias encountered in clinical practice. ${ }^{(1)}$ In developed countries, there has been a steady increase in the number of patients with AF. This increase is associated with an increase in life expectancy, an increase in the occurrence of pathology

*Corresponding author: Evgeniy S. Ovsyannikov, PhD. Associate Professor of the Department of Internal Medicine, Voronezh N.N. Burdenko State Medical University. Voronezh, Russia. E-mail: ovses@yandex.ru of the cardiovascular system, and an improvement in the detection of latent forms of arrhythmia. At the same time, despite significant progress in diagnosis and treatment, this pathology remains one of the main causes of stroke, heart failure (HF), sudden cardiac death, and premature dementia.

There are three main mechanisms for the appearance and progression of AF: genetic predisposition, arrhythmogenic cardiomyopathy and structural myocardial remission in various heart pathologies. ${ }^{(2,3)}$ The course of AF and its form depend on the prevalence of one of these mechanisms over others. In most patients with a paroxysmal or persistent form of AF, the role of genetic factors in the development of arrhythmia is 
small. The progression of arrhythmias is associated with age and the presence of cardiovascular disease. In patients with persistent AF, the transformation of arrhythmias into more stable forms is determined by the presence of cardiovascular predictors of progression. ${ }^{(2)}$

It has been found that the presence of chronic heart failure increases the risk of AF by 5 times. ${ }^{(4)}$ The occurrence of AF in patients with heart failure is associated with atrial fibrosis, which is a hallmark of arrhythmogenic remodeling. Atrial fibrosis triggers are the activation of the renin-angiotensinaldosterone system, inflammation, and oxidative stress..$^{(5-8)}$ Furthermore, with an increase in the severity of symptoms of heart failure, the incidence and severity of AF increases.

To date, markers of AF progression are not clearly defined. In this regard, it is relevant and reasonable to identify such markers in patients with HF.

The aim of this research was to study the prognostic value of the 6-minute walk test (6MWT), NT-proBNP level, and left ventricular diastolic dysfunction (LVDD) for $\mathrm{AF}$ progression in patients with CHF.

\section{Materials and Methods}

The study was open-label, prospective and observational. It involved 96 participants with stable Class II and III CHF (the NYHA Functional Classification), who were included in the regional registry of CHF patients in September-November 2014. The diagnosis of CHF was made on the basis of the "ESC Recommendation for the Diagnosis and Treatment of Acute and Chronic Heart Failure," developed by the Working Group of European Society of Cardiology (ESC) for the Diagnosis and Treatment of Acute and Chronic HF with the participation of the Heart Failure Association as part of the ESC.

The 6MWT was used to measure the functional status of patients. The average follow-up was $35 \pm 2$ months and included a routine examination by the researcher every 3 months, telephone contacts with patients in case of a heartbeat/ interruption with ECG recording, and annual general clinical and laboratory instrumental examination: ECG, 24-hour ECG monitoring, echocardiography. AF progression was assessed by the results of 24-hour ECG monitoring and patient diary data. The appearance of a long-term, persistent (up to 1 year) or persistent form of AF was considered as a progression of arrhythmia.

All patients underwent a standard examination, which included clinical, laboratory and instrumental methods. Exercise tolerance (ET) was determined by the 6MWT. In order to assess ET, a complex of cardiorespiratory analysis was used to carry out functional medical tests, recording the distance in 6MWT, pulse oximetry, and heart rate. ${ }^{(9-11)}$ Laboratory methods included a general blood test, general urine analysis, and biochemical and enzyme-linked immunosorbent assay for determining the level of NT-pro-BNP using the "sandwich" version of the solid-phase ELISA and reagent kits "NTproBNP-IFA-Best" (Vector Best, Russia) on the IMMULITE 2000 automatic analyzer (Siemens Diagnostics, USA). Measurements were performed at the time of inclusion in the study and annually during the observation time.
LVDD was evaluated using Doppler flow studies. Measurements of transmitral inflow included the peak early filling (E-wave) and late diastolic filling (A-wave) velocities $(\mathrm{cm} / \mathrm{s})$, the E/A ratio, deceleration time of the E-wave (ms), and isovolumetric relaxation time (IVRT) (ms).

The study was carried out in compliance with Ethical Principles for Medical Research Involving Human Subjects, Adopted by the 18th WMA General Assembly, Helsinki, Finland, June 1964, and amended by the 52nd WMA General Assembly, Edinburgh, Scotland, October 2000. The study was approved by the Ethics Committee of Voronezh State Medical University. Written informed consent was obtained from each patient.

All data was evaluated with STATGRAPHICS Plus 5.1 For descriptive analysis, results are presented as mean \pm standard deviation (SD), median (Me), and 95\% confidence interval $(95 \% \mathrm{CI})$. For data with normal distribution, inter-group comparisons were performed using Student's t-test. MannWhitney U test and Wilcoxon criterion were used to compare means of variables not normally distributed. Group comparisons with respect to categorical variables were performed using chisquare tests or, alternatively, Fisher's exact test when expected cell counts were less than 5. A probability value of $P<0.05$ was considered statistically significant.

\section{Results}

During the entire observation period, none of the patients were dropped out due to death. Cardiovascular complications were detected in 5(5.2\%) patients: myocardial infarction was diagnosed and confirmed by laboratory and instrumental methods in 4(4.1\%) patients, and acute cerebrovascular accident in $1(1 \%)$ of the patients. Arrhythmia progression was observed in $31(32.3 \%)$ patients. Two groups of patients were formed depending on the clinical course of AF. Group 1 included AF patients who did not show any progression of arrhythmia during the observation period $(n=65)$; Group 2 included patients with AF progression $(\mathrm{n}=31)$. Groups 1 and 2 were comparable in gender and age composition, the presence of arterial hypertension, coronary heart disease, diabetes mellitus, and chronic obstructive pulmonary disease. The proportions of patients who previously had myocardial infarction were also comparable in both groups.

The initial 6MWT distance reflects a statistically significantly lower ET in patients of Group 2 compared with patients of Group 1 (by $33 \mathrm{~m}, P=0.0159$ ) (Fig.1). At the time, in Groups 1 and 2 there was no significant difference in LVEF $(43.03 \pm 1.23 \%$ and $44.18 \pm 0.65 \%, P=0.08)$, LVESD $(4.11 \pm 0.07$ $\mathrm{cm}$ and $4.06 \pm 0.06 \mathrm{~cm}, P=0.46)$, LVEDD $(5.63 \pm 0.09 \mathrm{~cm}$ and $5.25 \pm 0.05 \mathrm{~cm}, P=0,77)$, the size of $\operatorname{LA}(4.51 \pm 0.2 \mathrm{~cm}$ and $4.46 \pm 0.14 \mathrm{~cm}, P=0.32)$, LVPWT $(1.24 \pm 0.05 \mathrm{~cm}$ and $1.27 \pm 0.02$ $\mathrm{cm}, P=0.71)$, and IVST $(1.19 \pm 0.03 \mathrm{~cm}$ and $1.24 \pm 0.02 \mathrm{~cm}$, $P=0.09$ ).

The analysis of the initial parameters of LVDD in Groups 1 and 2 showed that LVDD type I was found in $80.6 \%$ and $23 \%$, respectively $(P=0.013)$, LVDD type II in $19.4 \%$ and $70.7 \%$, respectively $(P=0.008)$, and LVDD type III in 0 and $6 \%$, respectively $(P=0.024)$. 


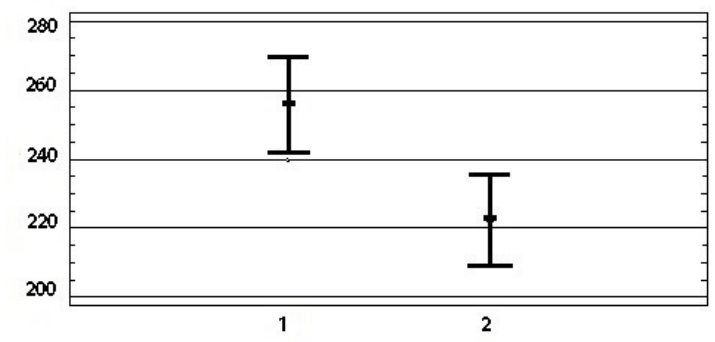

Fig. 1. The initial 6MWT distance $(m)$ in Groups 1 and $2(P=0.0159)$.

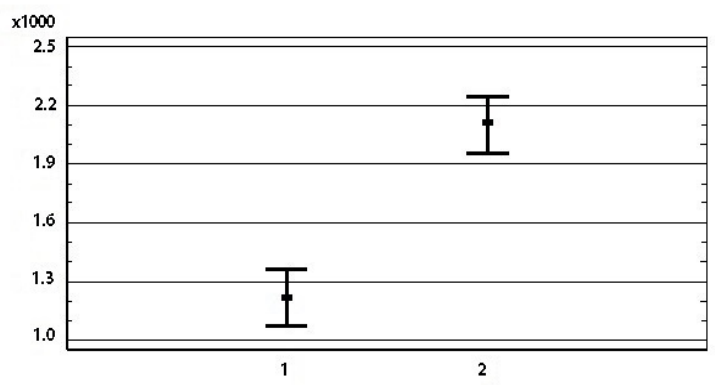

Fig. 2. The initial Nt-proBNP level (pg/ml) in Groups 1 and $2(P<0.001)$.

The initial Nt-proBNP level in Group 1 was 1.7 times lower than in Group $2(P<0.001)$ (Fig. 2).

\section{Discussion}

Thus, the data obtained showed a higher Nt-proBNP level in the group of patients with progressive AF compared with the group of patients with a stable course of arrhythmia. The higher initial Nt-proBNP level in these patients can be explained by the following structural and functional changes in the myocardium: a higher level of myocardial fibrosis and impaired relaxation of cardiomyocytes, which caused arrhythmogenic remodeling of the myocardium of the LA and $\mathrm{AF}$ progression (i.e. the transition of paroxysmal $\mathrm{AF}$ into more stable forms of arrhythmia - persistent and permanent).

In CHF patients, a statistically significant effect on the progression of arrhythmias, in addition to an increase in the Nt-proBNP level, was exerted by the following factors: impaired myocardial relaxation and decreased 6MWT distance (reduced ET). Impairment of the diastolic function of the left ventricular myocardium in patients with arrhythmia progression, revealed upon inclusion of patients in the study, could be the result of hypertrophy and/or decreased elasticity of the cardiomyocytes/myocardium. Since the frequency of arterial hypertension was comparable in the study groups, and the initial echocardiography data did not show a statistically significant difference in the values of LVPWT and IVST, it can be assumed that it was the decrease in myocardial elasticity (fibrosis) that made the most important contribution to the progression of AF into more stable forms. Impairment of $\mathrm{LV}$ myocardial relaxation leads to a redistribution of the transmitral blood flow: Most of the blood enters the LV during the LA systole. This causes an increase in the load on LA cardiomyocytes, which is accompanied by a compensatory increase in the filling pressure and subsequent structural reorganization of the LA, which, in our opinion, also contributed to the progression of AF and lower initial results of 6MWT.

The results of our study are consistent with data from other authors, in particular, a retrospective analysis of patients who were included in the BOREAS-CAG registry. In that study, it was found that an increased Nt-proBNP level in patients with coronary artery disease was the main factor predisposing to the development of new cases of AF and the development of heart failure. ${ }^{(12)}$

Thus, in patients with CHF, an increase in the level of Nt-proBNP, impaired myocardial relaxation, and a decrease in 6MWT distance may serve as predictors of AF progression with the transition of arrhythmias to stable forms.

\section{Competing Interests} interests.

The authors declare that they have no competing

\section{Disclaimers}

The opinions expressed in this article are the authors' own and do not reflect the view of the institutions or funder.

\section{References}

1. Kirchhof P, Benussi S, Kotecha D, Ahlsson A, Atar D, Casadei B, et al.; ESC Scientific Document Group.2016 ESC Guidelines for the management of atrial fibrillation developed in collaboration with EACTS. Eur Heart J. 2016;37(38):28932962. doi: 10.1093/eurheartj/ehw210.

2. Heijman J, Voigt N, Nattel S, Dobrev D. Cellular and molecular electrophysiology of atrial fibrillation initiation, maintenance, and progression. Circ Res. 2014;114(9):148399. doi:10.1161/CIRCRESAHA.114.302226.

3. Tokmachev RE, Mukhortova MS, Budnevsky AV, Tokmachev EV, Ovsyannikov ES. [Comorbidity of chronic heart failure and chronic obstructive pulmonary disease: features of pathogenesis, clinic and diagnosis]. Cardiovascular Therapy and Prevention. 2018;17(6):62-68. [Article in Russian].

4. Wang TJ, Larson MG, Levy D, Vasan RS, Leip EP, Wolf PA, et al. Temporal relations of atrial fibrillation and congestive heart failure and their joint influence on mortality: the Framingham Heart Study. Circulation. 2003;107(23):2920-5.

5. Burstein B, Nattel S. Atrial fibrosis: mechanisms and clinical relevance in atrial fibrillation. J Am Coll Cardiol. 2008;51(8):802-9. doi: 10.1016/j.jacc.2007.09.064.

6. Drobysheva ES, Tokmachev RE, Budnevsky AV, Kravchenko AYa. [Predictive value of markers of cardiac cachexia in chronic heart failure]. Cardiovascular Therapy and Prevention. 2016;15(4):80-83. [Article in Russian].

7. Tokmachev RE, Budnevsky AV, Kravchenko AY. [The role of inflammation in the pathogenesis of chronic heart failure]. Ter Arkh. 2016;88(9):106-110. doi: 10.17116/ terarkh2016889106-110. [Article in Russian].

8. Budnevsky AV, Shurupova AD, Kravchenko AY, 
Tokmachev RE. [Clinical efficacy of acute respiratory viral infections prevention in patients with chronic heart failure]. Ter Arkh. 2019;91(3):36-41. doi: 10.26442/00403660.2019.0 3.000111. [Article in Russian].

9. Tokmachev RE, Maksimov AV, Budnevsky AV, Batishcheva GA, Ovsyannikov ES, Kravchenko AYa, Kurgalin SD. A device for cardiorespiratory analysis and a method for evaluating a cardiorespiratory state. Patent for invention RUS No. 2637917. Application No. 2016148274 dated December 9, 2016. Publ. 12/07/2017.

10. Tokmachev RE, Budnevsky AV, Kravchenko AYa. The possibility of non-pharmacological methods in increasing clinical efficiency of treating patients with chronic heart failure and metabolic syndrome. Research Journal of Pharmaceutical, Biological and Chemical Sciences. 2017;8(6):832-839.

11. Shiryaev OYu, Yankovskaya VL, Budnevsky AV, Ovsyannikov ES. Psychosomatic aspects of congestive heart failure. International Journal of Biomedicine. 2017;7(3):248250.

12. Murakami N, Tanno M, Kokubu N, Nishida J, Nagano $\mathrm{N}$, Ohnishi $\mathrm{H}$, et al. Distinct risk factors of atrial fibrillation in patients with and without coronary artery disease: a crosssectional analysis of the BOREAS-CAG Registry data.Open Heart. 2017;4(1):e000573. doi: 10.1136/openhrt-2016-000573. 